\title{
Rapid Degradation of the Electrical Properties of 2D MoS 2 Thin Films under Long-Term Ambient Exposure
}

\author{
Bhim Chamlagain* and Saiful I. Khondaker*
}

Cite This: ACS Omega 2021, 6, 24075-24081

Read Online

ABSTRACT: The $\mathrm{MoS}_{2}$ thin film has attracted a lot of attention due to its potential applications in flexible electronics, sensors, catalysis, and heterostructures. Understanding the effect of long-term ambient exposure on the electrical properties of the thin film is important for achieving many overreaching goals of this material. Here, we report for the first time a systematic study of electrical property variation and stability of $\mathrm{MoS}_{2}$ thin films under ambient exposure of up to a year. The $\mathrm{MoS}_{2}$ thin films were grown via the sulfurization of $6 \mathrm{~nm}$ thick molybdenum films. We found that the resistance of the samples increases by $114 \%$ just in 4 weeks and $430 \%$ in 4 months and they become fully insulated in a year of ambient exposure. The dual-sweep current-voltage $(I-V)$ characteristic shows hysteretic behavior for a 4-month-old sample which further exhibits pronounced nonlinear $I-V$ curves and hysteretic behavior after 8 months. The X-ray photoelectron
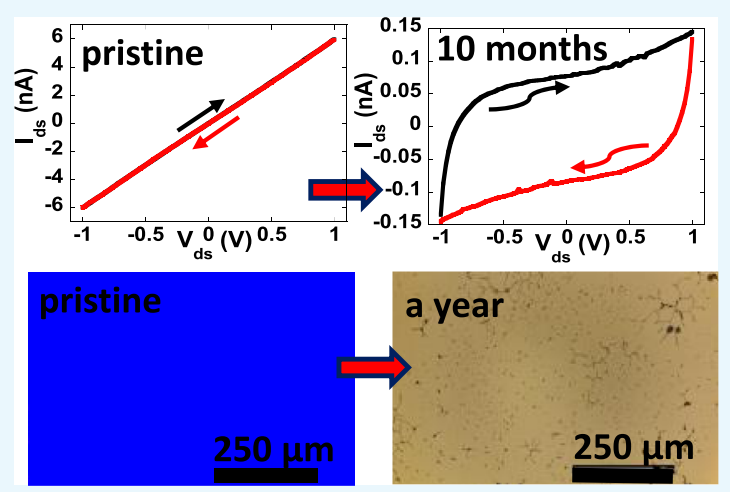
spectroscopy measurements show that the $\mathrm{MoS}_{2}$ thin film gradually oxidizes and $13.1 \%$ of $\mathrm{MoO}_{3}$ and $11.8 \%$ oxide of sulfur were formed in 4 months, which further increased to 23.1 and $12.7 \%$ in a year, respectively. The oxide of the sulfur peak was not reported in any previous stability studies of exfoliated and chemical vapor deposition-grown $\mathrm{MoS}_{2}$, suggesting that the origin of this peak is related to the distinct crystallinity of the $\mathrm{MoS}_{2}$ thin film due to its smaller grain sizes, abundant grain boundaries, and exposed edges. Raman studies show the broadening of $E_{2 g}^{1}$ and $A_{1 g}$ peaks with increasing exposure time, suggesting an increase in the disorder in $\mathrm{MoS}_{2}$. It is also found that coating the $\mathrm{MoS}_{2}$ thin film with polymethylmethacrylate can effectively prevent the electrical property degradation, showing only a $6 \%$ increase in resistance in 4 months and $40 \%$ over a year of ambient exposure.

\section{INTRODUCTION}

Thin films of two-dimensional (2D) transition-metal dichalcogenides (TMDs) prepared via the chalcogenization of metal and metal oxide films have attracted significant research interest due to their uniform large-area coverage, controlled synthesis in a wide range of thicknesses, and cost-effective relatively easier synthesis process. ${ }^{1-8}$ This growth method not only provides control over the thickness of the TMD films but also transitions from horizontal to vertical orientation of the TMD sheet with the initial thickness of the metal film used. ${ }^{5,9}$ The large-area $\mathrm{MoS}_{2}$ thin film prepared via the sulfurization of molybdenum (Mo) films has been demonstrated as a potential candidate for catalysis applications due to its abundant exposed edges in vertically oriented sheets. ${ }^{6}$ Vertical and lateral heterojunctions along with their application in photodetection were also demonstrated. ${ }^{10,11}$ Recently, the film has been investigated for possible applications in flexible electronics and gas sensing. ${ }^{12,13}$ However, TMD films have much smaller grain sizes, typically in the range of $10-100 \mathrm{~nm}$, which give rise to significantly more grain boundaries than their exfoliated and coevaporation chemical vapor deposition (CVD)-based counterparts. ${ }^{8}$ Furthermore, the vertical sheet of TMDs has abundant exposed edges which could be prone to ambient gases and moisture. The interaction of ambient gases and moisture with the thin film could pose major challenges in not only the practical realization of the thin-film devices but also the reproducibility of the results.

Although a few studies of the stability of mechanically exfoliated and coevaporation CVD-based 2D $\mathrm{MoS}_{2}$ under ambient exposure have been reported mainly focusing on structural and optical property variation, ${ }^{14-19}$ the study of the stability of the $\mathrm{MoS}_{2}$ thin film has not been reported to date. In addition, a systematic study of the electrical property variation of $\mathrm{MoS}_{2}$ grown by any technique has not been investigated. For CVD-grown monolayer $\mathrm{MoS}_{2}$, oxidation at grain boundaries and sulfur $(S)$ vacancies has been reported with the progressive oxidation of the film over time. ${ }^{14}$ In this film, grain boundaries have primarily exposed Mo sites, which promote the formation of $\mathrm{MoO}_{3}$. Theoretical calculations also showed the pronounced

Received: July 5, 2021

Published: September 9, 2021 
oxidation and absorption of the atmospheric gases at the defect centers and grain boundaries. ${ }^{20-22}$ These findings suggest that TMDs with abundant grain boundaries are more susceptible to atmospheric oxygen and moisture. In another study, it has been reported that decreasing the $S$ vacancy density by adjusting the growth conditions of CVD $\mathrm{MoS}_{2}$ monolayers could slow the degradation, ${ }^{19}$ suggesting that defects and grain boundaries in the film are crucial for the film degradation. In contrast to exfoliated and CVD-based $\mathrm{MoS}_{2}$, the thin film has distinct crystallinity, which has abundant grain boundaries and edges in the film. ${ }^{8}$ Therefore, it is critical to investigate the long-term stability of the $\mathrm{MoS}_{2}$ thin film under ambient exposure. In addition, since $\mathrm{MoS}_{2}$ is an electronic material with many potential applications in electronics and optoelectronics, it is important to systematically study the effect of long-term ambient exposure on the electronic properties of $\mathrm{MoS}_{2}$.

Here, we present a systematic study on the variation of electrical properties of the large-area $\mathrm{MoS}_{2}$ thin film prepared via the sulfurization of Mo films by keeping it under ambient conditions. We observed that the resistance of $\mathrm{MoS}_{2}$ devices monotonically increases with time and the average resistance of the devices increases by $114 \%$ in just 4 weeks, which further increases by $430 \%$ in 4 months and the samples become completely insulated in a year. In addition, the dual-sweep current-voltage $(I-V)$ characteristic becomes nonlinear and shows hysteretic behavior after 4 months, which becomes pronounced with increasing exposure time. X-ray photoelectron spectroscopy (XPS) measurements show that the formation of the oxides of Mo and S due to the oxidation of the $\mathrm{MoS}_{2}$ film gradually increases with time. The oxide of the sulfur peak was not reported in any previous stability studies of exfoliated or CVD-grown $\mathrm{MoS}_{2}$, suggesting that the origin of this peak is related to the distinct crystallinity of the film due to its smaller grain sizes and orientation of the sheet. We observed a shift of Mo 3d and S 2p XPS doublet peaks to lower binding energies (BEs) for aged $\mathrm{MoS}_{2}$ films, suggesting a relative shift of the Fermi level toward the valence band edge, consistent with electrical transport measurement data. In addition, XPS data also showed that organic contaminants and moisture are absorbed on the aged $\mathrm{MoS}_{2}$ films, which suggest that the localized electrons at the $\mathrm{MoO}_{3}$ sites promote the absorption of polar molecules and moisture from the atmosphere, resulting in the observed hysteresis effect in the $I-V$ curves. Raman studies showed an increase of full width at half-maximum (fwhm) of $E_{2 g}^{1}$ and $A_{1 g}$ peaks with increasing exposure time, suggesting an increase in the disorder in $\mathrm{MoS}_{2}$ films. Our study suggests that the $\mathrm{MoS}_{2}$ film actively interacts with atmospheric oxygen to gradually increase $\mathrm{MoO}_{3}$ and oxide of the sulfur amount in the film, which increases the resistance of the film and eventually becomes electrically insulated in a year. We also found that coating the $\mathrm{MoS}_{2}$ thin film with polymethylmethacrylate (PMMA) can effectively prevent the electrical property degradation, showing only a $6 \%$ increase in resistance in 4 months and $40 \%$ over a year of ambient exposure. Our study reported here on the long-term stability, especially the variation of electrical properties of the $\mathrm{MoS}_{2}$ thin film is an important step forward in achieving the overreaching goals of $\mathrm{MoS}_{2}$ thin films in practical applications.

\section{RESULTS AND DISCUSSION}

Figure 1a shows a digital image of a $6 \mathrm{~nm}$ thick Mo film, while Figure $1 \mathrm{~b}$ shows a digital image of the same film after sulfurization. The change of color indicates that the Mo film
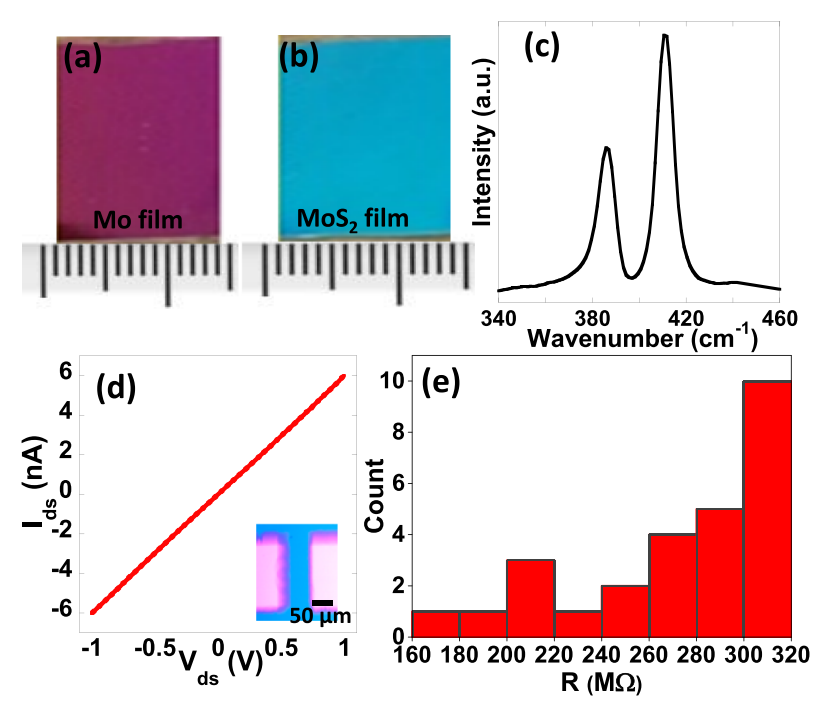

Figure 1. Digital image of the (a) molybdenum film, (b) $\mathrm{MoS}_{2}$ film after the sulfurization of the Mo film, (c) Raman spectrum of the pristine $\mathrm{MoS}_{2}$ thin film, (d) current-voltage $(I-V)$ characteristics of a representative pristine $\mathrm{MoS}_{2}$ device, and (e) histogram of resistance for the pristine $\mathrm{MoS}_{2}$ devices.

was successfully sulfurized to form the $\mathrm{MoS}_{2}$ thin film. Raman spectra of the sulfurized film presented in Figure 1c show two prominent peaks at 386.2 and $411.0 \mathrm{~cm}^{-1}$, corresponding to the in-plane $E_{2 g}^{1}$ and out-of-plane $A_{1 g}$ vibrational modes of $\mathrm{MoS}_{2}$, respectively, with a position difference of $24.8 \mathrm{~cm}^{-1}$, indicating the formation of the multilayer $\mathrm{MoS}_{2}$ film. ${ }^{23-27}$ For electrical transport measurements, $\mathrm{MoS}_{2}$ devices of $100 \mu \mathrm{m}$ channel length and $300 \mu \mathrm{m}$ channel width were fabricated by depositing $5 \mathrm{~nm} / 35 \mathrm{~nm}$ of $\mathrm{Cr} / \mathrm{Au}$ using a shadow mask. Figure $1 \mathrm{~d}$ shows the current-voltage $(I-V)$ characteristics of a representative pristine $\mathrm{MoS}_{2}$ device. The resistance calculated from the linear region of the $I-V$ curve was found to be $168 \mathrm{M} \Omega$. We have measured a total of $27 \mathrm{MoS}_{2}$ devices that were fabricated on the same chip, and the resistance of the devices varies from 165 to $318 \mathrm{M} \Omega$ with an average value of $266 \mathrm{M} \Omega$ (presented in Figure 1e and Supporting Information, Figure S1). The device-todevice variation of resistance is not due to the variation of the thickness of the films as we have measured the thickness of the Mo film at different locations and found the thickness to be uniform. In addition, all the devices have the same dimensions. Therefore, the device-to-device resistance variation could be due to the local inhomogeneity of the $\mathrm{MoS}_{2}$ film resulting from the growth process. These values of resistance are within the range of reported resistance values of the $\mathrm{MoS}_{2}$ film prepared by similar methods. ${ }^{9,10,12,28-30}$

The devices were then left exposed under atmospheric conditions (lab temperature, pressure, and humidity) for a period of a year during which their electrical characterizations were periodically measured. Figure 2 a shows the $I-V$ characteristics of the $\mathrm{MoS}_{2}$ device presented in Figure 1d measured every week for up to 6 weeks and then at 9, 12, and 16 weeks. The calculated values of resistance of this device with time are presented in Figure $2 \mathrm{~b}$. The resistance of the device increases monotonically with time. After 1 week, the resistance increased to $193 \mathrm{M} \Omega$, an increase of $14 \%$. The resistance continued to increase by $61 \%$ in 2 weeks, $92 \%$ in 3 weeks, $114 \%$ in 4 weeks, $164 \%$ in 5 weeks, $175 \%$ in 6 weeks, $316 \%$ in 9 weeks, $415 \%$ in 12 weeks, and $508 \%$ in 16 weeks. We have measured all the 27 $\mathrm{MoS}_{2}$ devices after 4 months, and an increase of the resistance 

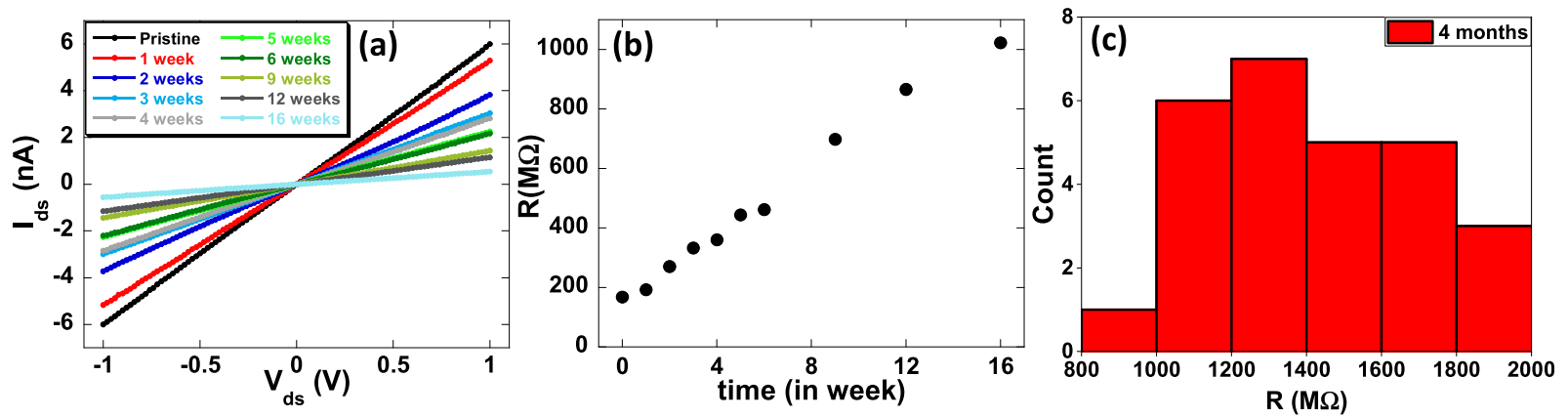

Figure 2. (a) $I-V$ characteristics of the $\mathrm{MoS}_{2}$ device (presented in Figure 1d) measured every week for up to 6 weeks and then at 9, 12, and 16 weeks. (b) Calculated values of the resistance of the device with time and (c) histogram of resistance for 27 devices on a chip measured after 4 months.

was observed for every sample (Figure $2 \mathrm{c}$ and Supporting Information, Figure S1). The resistance of the 4-month-old samples varies from 976 to $1990 \mathrm{M} \Omega$ with an average value of $1405 \mathrm{M} \Omega$, which is a $\sim 430 \%$ increase compared to the average resistance of the pristine sample. While there are no reports on the electrical property variation of $\mathrm{MoS}_{2}$ thin films due to longterm ambient exposure, there is only one report of the electrical property variation of the CVD-grown $\mathrm{MoS}_{2}$ monolayer, which showed that the drain current was decreased by up to 2 orders of magnitude after a month of ambient exposure. ${ }^{14}$ Assuming that the reported current in the CVD-grown monolayer is Ohmic, this would mean that the resistance variation in the CVD-grown $\mathrm{MoS}_{2}$ monolayer is faster than that in the thin-film sample. This is quite surprising given that thin-film $\mathrm{MoS}_{2}$ has smaller grain sizes with significantly more grain boundaries compared to the CVD-grown monolayer. However, we note that the reported CVD-grown $\mathrm{MoS}_{2}$ device was fabricated after 3 months by keeping the sample in marginal vacuum which makes the comparison complicated. Since the thickness of the $\mathrm{MoS}_{2}$ thin film is $\sim 14 \mathrm{~nm}$, which is $\sim 20$ times higher than that of the CVDgrown $\mathrm{MoS}_{2}$, one could argue that in the thin film, upper layers could protect the lower layers from ambient effects, which might explain the less dramatic electrical property variation for a 1month-old sample; however, we note that a 1-year-old sample is completely insulated, suggesting that every layer is affected by ambient exposure and top layers do not protect the bottom layers.

We also studied the hysteresis effect on the $I-V$ curves to gain more insights into the electrical property variation by measuring dual-sweep (forward and reverse) $I-V$ characteristics of the devices. This is presented in Figure 3, where we show $I-V$ curves of a representative device measured immediately after fabrication, 4, 8, and 10 months of ambient exposure. It is noted that the hysteresis behavior on the $I-V$ curve of the $\mathrm{MoS}_{2}$ sample due to aging has never been reported before. Figure $3 \mathrm{a}$ shows the $I-V$ curve for the pristine $\mathrm{MoS}_{2}$ sample measured from -1 to $1 \mathrm{~V}$ (forward) and then from 1 to $-1 \mathrm{~V}$ (reverse), while Figure $3 b-d$ represents $I-V$ curves of the same sample measured after 4, 8, and 10 months, respectively. The $I-V$ curve of the pristine device does not show any hysteretic behavior, while very little hysteresis was observed for the 4-month-old sample. However, significant hysteresis was observed for 8- and 10-month-old samples. The observed hysteretic behavior suggests the formation of trap charge states on the film for aged samples, which is originated from the ambient effect. ${ }^{31}$ Due to the nonlinear hysteretic nature of the $I-V$ curve, we did not calculate the resistance of samples that were more than 4-monthold. After a year, the sample became completely electrically
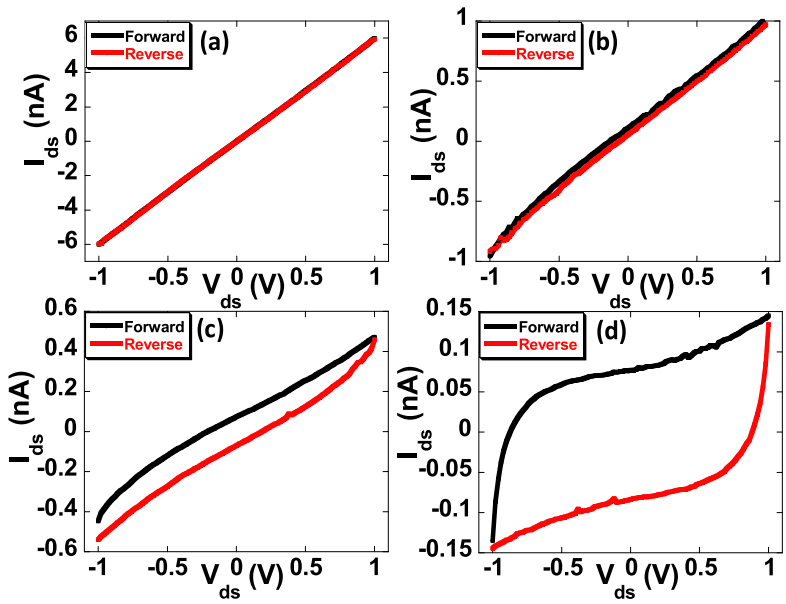

Figure 3. Dual-sweep $I-V$ characteristics of the $\mathrm{MoS}_{2}$ device measured (a) immediately after fabrication, (b) in 4 months, (c) in 8 months, and (d) in 10 months.

insulated. Interestingly, the film color also changed for a 1-yearold sample (Supporting Information, Figure S2) in comparison to the pristine film. We have attempted to measure gate dependence electrical properties by using highly doped $\mathrm{Si}$ as a back gate; however, no gate dependence was observed similar to what has been reported in a few other reports., $92,28,32$

To identify the chemical states of the film, which are responsible for the observed changes in electronic transport properties, we performed XPS measurements of the pristine, 4month-old (when a hysteretic $I-V$ curve appears), and 1-yearold (when the film becomes completely electrically insulated) $\mathrm{MoS}_{2}$ films (Figure 4). The symbols represent experimental data and the solid lines represent convoluted spectra. Figure 4a shows the Mo 3d core-level XPS spectra of the pristine $\mathrm{MoS}_{2}$ with three prominent peaks expected at 226.0, 229.1, and $232.2 \mathrm{eV}$ corresponding to the BEs of S $2 \mathrm{~s}, \mathrm{Mo}^{4+} 3 \mathrm{~d}_{5 / 2}$, and $\mathrm{Mo}^{4+} 3 \mathrm{~d}_{3 / 2}$ electrons, respectively. ${ }^{32,33}$ Figure $4 \mathrm{~d}$ shows the $\mathrm{S} 2 \mathrm{p}$ core-level XPS spectra of the same sample with peak positions at 161.7 and $162.8 \mathrm{eV}$ corresponding to $\mathrm{S}^{2+} 2 \mathrm{p}_{3 / 2}$ and $\mathrm{S}^{2+} 2 \mathrm{p}_{1 / 2}$ spin-orbit split components of $\mathrm{MoS}_{2}$, respectively. ${ }^{30,33,34}$ For the 4-monthold $\mathrm{MoS}_{2}$ film, the Mo 3d XPS spectra (Figure 4b) show a slight shift to lower BEs. In addition, two new peaks were observed at 232.8 and $235.2 \mathrm{eV}$, which correspond to $\mathrm{Mo}^{6+}$ peaks due to the formation of $\mathrm{MoO}_{3} .{ }^{32,33,35}$ The S 2p XPS spectra (Figure 4e) of the same sample also show a slight shift to lower BEs, and a new peak appeared at $168.0 \mathrm{eV}$, which belongs to $S^{6+}$ due to the oxidation of $S .{ }^{33,35}$ The downshift of BEs indicates a relative shift of the Fermi level toward the valence band edge, suggesting the 

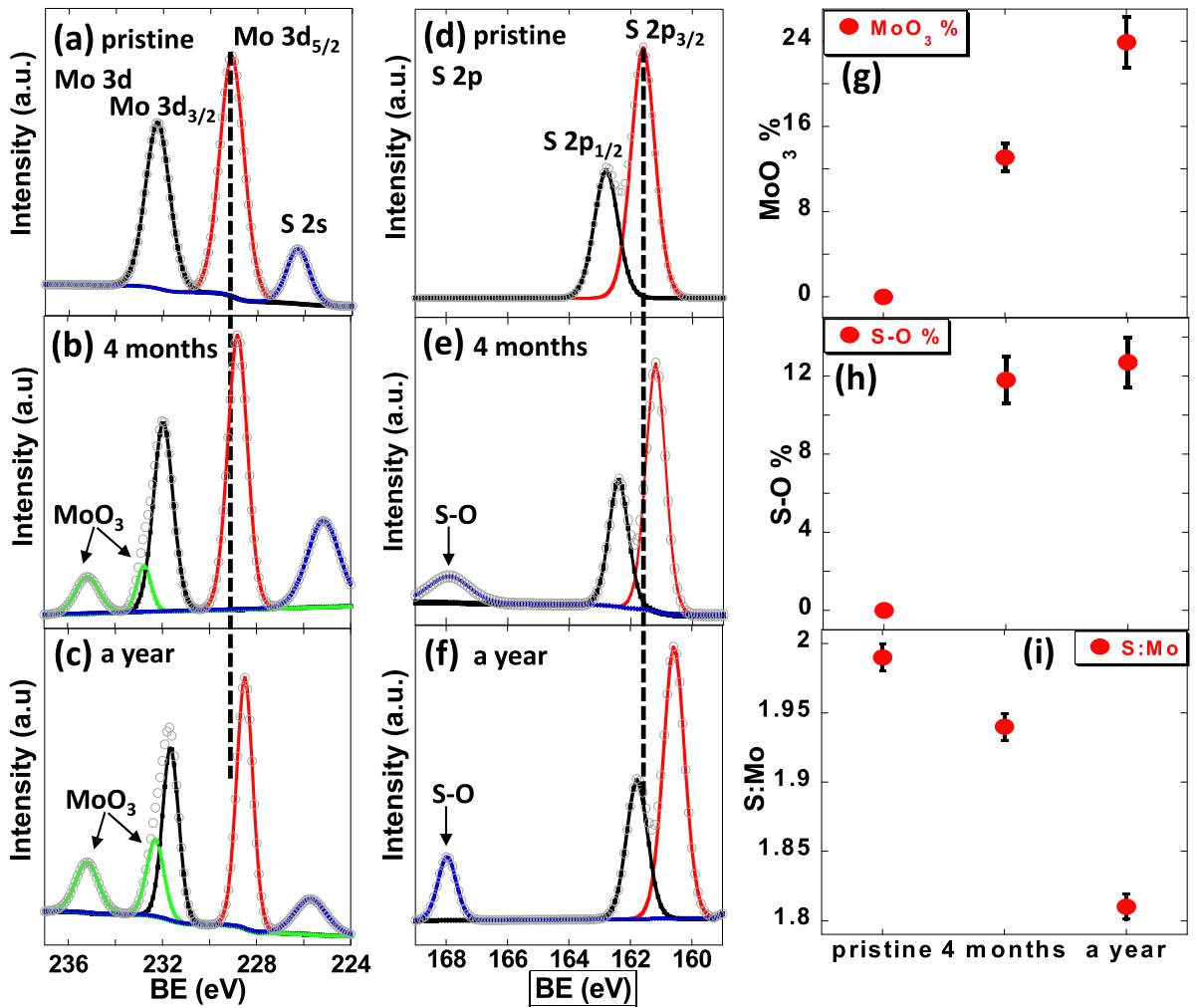

Figure 4. XPS spectra of the $\mathrm{MoS}_{2}$ thin film. Mo 3d core-level XPS spectra of the (a) pristine $\mathrm{MoS}_{2}$ film, (b) 4-month-old film, and (c) 1-year-old film. S $2 \mathrm{p}$ core-level XPS spectra of the (d) pristine $\mathrm{MoS}_{2}$ film, (e) 4-month-old film, and (f) 1-year-old film. The spectra were deconvoluted using GaussianLorentzian curves. The symbols are the experimental points, and the solid lines are the deconvolution of the data. $(\mathrm{g})$ Percentage of $\mathrm{MoS}_{2}$ transformed into $\mathrm{MoO}_{3}$ with time, (h) percentage of $\mathrm{MoS}_{2}$ transformed into the oxide of sulfur with time, and (i) variation of the S/Mo ratio with time.

p-doping of $\mathrm{MoS}_{2}$ films due to oxidation, ${ }^{33}$ which is consistent with electrical transport measurements. Similarly, the XPS spectra of Mo and S for the 1-year-old $\mathrm{MoS}_{2}$ film are presented in Figure $4 c, f$, respectively. For this sample, all the peaks corresponding to Mo $3 \mathrm{~d}, \mathrm{~S} 2 \mathrm{~s}$, and S $2 \mathrm{p}$ were observed along with additional peaks at 232.4 and 235.2 corresponding to $\mathrm{Mo}^{6+}$ and at $168.0 \mathrm{eV}$ corresponding to $\mathrm{S}^{6+}$. Interestingly, $\mathrm{Mo}^{6+}$ peaks of a 1-year-old sample became more prominent than the 4 month-old-sample, while the $S^{6+}$ peak remained almost the same, which suggests that more $\mathrm{MoS}_{2}$ is converted into $\mathrm{MoO}_{3}$, while the oxide of sulfur remains almost constant.

The percentages of the $\mathrm{MoO}_{3}$ formation and oxide of $\mathrm{S}$ are shown in Figure 4g,h, respectively. Remarkably, $13.1 \% \mathrm{MoS}_{2}$ converted to $\mathrm{MoO}_{3}$ in 4 months, which increased to $23.1 \%$ in a year. On the other hand, $11.8 \%$ of oxide of $S$ was calculated for the 4-month-old film, which slightly increased to $12.7 \%$ for a 1 year-old film (Figure 4h). The S/Mo ratio for the pristine $\mathrm{MoS}_{2}$ film was 1.99:1, which decreased to 1.94:1 for the 4-month-old sample and further decreased to 1.81:1 for a 1-year-old sample (Figure $4 \mathrm{i}$ ). Although the observed $13.1 \%$ of $\mathrm{MoO}_{3}$ in the 4month-old thin-film sample is comparable to reported $\mathrm{MoS}_{2}$ conversion into $\mathrm{MoO}_{3}$ (14.4\%) for the CVD-grown monolayer in 6 months, ${ }^{14}$ the formation of the oxide of $S$ was not observed for any previous stability studies of exfoliated or CVD-grown $\mathrm{MoS}_{2}$. However, the formation of the oxide of $S$ was observed in the oxygen plasma-treated $\mathrm{MoS}_{2}$ thin film prepared by the same method, ${ }^{33}$ suggesting that the origin of this peak is related to the distinct crystallinity of the film originated from its smaller grain sizes, abundant grain boundaries, and exposed edges. These results suggest that the $\mathrm{MoS}_{2}$ thin film leads to the incorporation of atmospheric oxygen with Mo and $S$ for the simultaneous formation of their oxides. Since the formation of $\mathrm{MoO}_{3}$ and oxide of $S$ in the film is due to the absorption of oxygen from the atmosphere, the overall oxidation of the $\mathrm{MoS}_{2}$ film is faster than the oxidation of CVD-grown monolayer $\mathrm{MoS}_{2}$. In addition, organic contaminants and water were also detected on the $\mathrm{MoS}_{2}$ film (Supporting Information, Figures S3 and S4). This result suggests that the localized electrons at $\mathrm{MoO}_{3}$ domains attract the polar molecules and moisture from the atmosphere, which is the origin of the observed hysteresis in $I-V$ curves in electrical transport measurements. We rule out the degradation of contact as a possible mechanism for the observed increase of resistance in the aged samples. This is due to the fact that the $\mathrm{MoS}_{2}$ film underneath the metal contacts is expected to protect the film from interaction with the atmospheric gases (similar to how PMMA protects the $\mathrm{MoS}_{2}$ film discussed in the next section). As a result, the contact resistance is expected to remain the same with time. Previous morphological studies on the ambientexposed CVD-grown $\mathrm{MoS}_{2}$ monolayer and exfoliated flakes showed cracks and pits. ${ }^{14-16,19}$ In our morphological studies using atomic force microscopy (AFM) and scanning electron microscopy (SEM) (Supporting Information, Figure S5), we did not observe any visible cracks in the $\mathrm{MoS}_{2}$ thin film after longterm ambient exposure. However, we observed an increase in surface roughness with time along with dot- and dendrite-like structures, which can be explained due to the oxidation of the film, the absorbance of organic contaminants, and moisture. ${ }^{16}$ These observations are consistent with the observed increase of resistance and hysteresis for the aged devices.

To further investigate the ambient effects on the $\mathrm{MoS}_{2}$ film, Raman spectra of the $\mathrm{MoS}_{2}$ samples were recorded after ambient exposure. The measured Raman spectra of 4-month- and 1-year- 
old samples are shown in Figure 5a. Raman spectra of the pristine sample are also presented in the same plot for

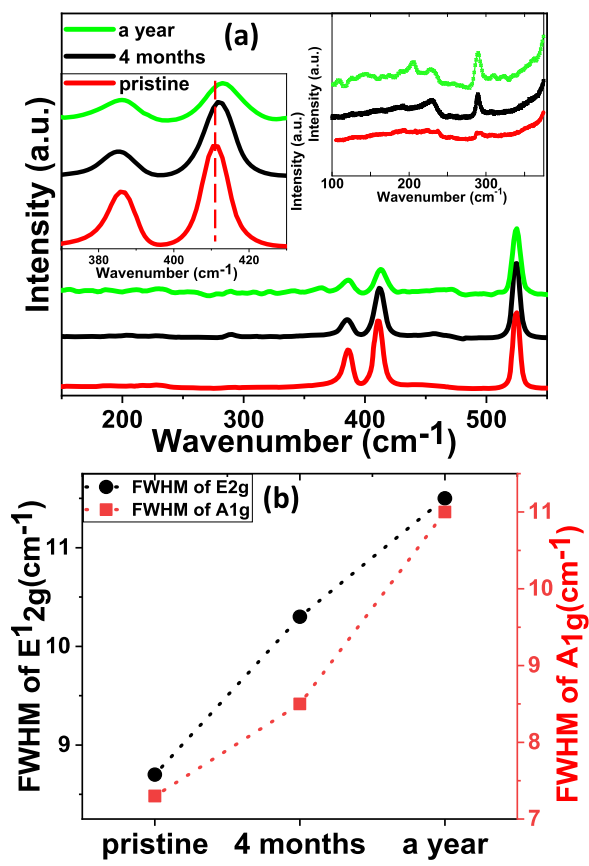

Figure 5. (a) Raman spectra of pristine, 4-month-old, and 1-year-old $\mathrm{MoS}_{2}$ films. The inset shows zoom-in Raman spectra of $\mathrm{MoS}_{2} \mathrm{E}_{2 \mathrm{~g}}^{1}$ and $A_{1 g}$ peaks and spectra at the low-wavenumber range. (b) fwhm of $E_{2 g}^{1}$ and $A_{1 g}$ peaks with an ambient exposure time.

comparison (red curve of Figure 5a). As in the pristine film, two $\mathrm{MoS}_{2}$ characteristic peaks were observed for the 4-monthold sample at 385.8 and $412.3 \mathrm{~cm}^{-1}$ corresponding to the inplane $E_{2 g}^{1}$ and out-of-plane $A_{1 g}$ vibrational modes. For the 4month-old sample, the $A_{1 \mathrm{~g}}$ peak was blue-shifted by $1.3 \mathrm{~cm}^{-1}$ compared with that of the pristine sample, suggesting that the pdoping of the $\mathrm{MoS}_{2}$ film further supports the results of XPS and electrical transport measurements. ${ }^{36}$ Similarly, $\mathrm{E}_{2 \mathrm{~g}}{ }^{1}$ and $\mathrm{A}_{1 \mathrm{~g}}$ peaks of a 1-year-old sample were observed at 385.6 and 412.8 $\mathrm{cm}^{-1}$ with an additional blue shift of the $A_{1 \mathrm{~g}}$ peak by $0.5 \mathrm{~cm}^{-1}$. In addition, we observed the broadening of Raman peaks of the films after ambient exposure. The fwhm of the pristine $\mathrm{MoS}_{2}$ sample was measured to be 7.3 and $8.7 \mathrm{~cm}^{-1}$ for $A_{1 \mathrm{~g}}$ and $E_{2 \mathrm{~g}}^{1}$ peaks, respectively, which increased to 8.5 and $10.3 \mathrm{~cm}^{-1}$ for the 4-month-old sample (Figure $5 \mathrm{~b}$ ). The fwhm of $\mathrm{A}_{1 \mathrm{~g}}$ and $\mathrm{E}_{2 \mathrm{~g}}^{1}$ peaks further increased to 11.0 and $11.5 \mathrm{~cm}^{-1}$ for a 1 -year-old sample, respectively. The observed broadening of Raman peaks of older $\mathrm{MoS}_{2}$ samples has been attributed to the disorder introduced in the $\mathrm{MoS}_{2}$ film due to the incorporation of oxygen. ${ }^{33}$ The inset of Figure 5a shows Raman spectra at the low-wavenumber region. For the 4-month-old sample, we observed prominent peak intensity at 224 and $285 \mathrm{~cm}^{-1}$, which was very weak for the pristine sample. For a 1-year-old sample, the same peaks were observed along with an additional peak at $201 \mathrm{~cm}^{-1}$. All these peak positions coincide with the $\mathrm{MoO}_{3}$ Raman peaks. ${ }^{37}$

To further confirm that the observed electrical property degradation is due to the ambient exposure, we encapsulated $\mathrm{MoS}_{2}$ thin-film devices with PMMA and measured electronic transport properties after 4 months and a year. We note that PMMA has been used to prevent the ambient degradation of extremely air-sensitive $2 \mathrm{D}$ materials. ${ }^{38,39}$ Figure 6 a shows the representative $I-V$ characteristics of pristine- and PMMA-
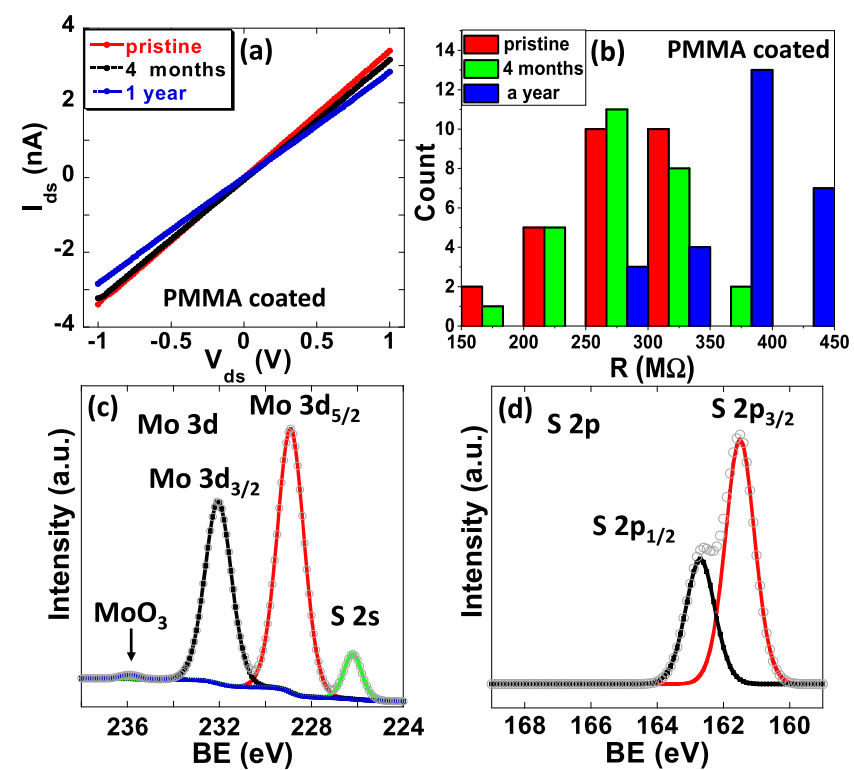

Figure 6. (a) Representative $I-V$ curve of a pristine $\mathrm{MoS}_{2}$, 4-monthold, and a 1-year-old PMMA-coated device. (b) Histogram of resistance for pristine- and PMMA-coated 4-month- and 1-year-old $\mathrm{MoS}_{2}$ devices. (c) Mo 3d and (d) S 2p core-level XPS spectra of the $\mathrm{MoS}_{2}$ film measured in a year, which was stored with PMMA coating. The symbols are the experimental points, and the solid lines are the deconvolution of the data using the Gaussian-Lorentzian fit.

covered 4-month- and 1-year-old $\mathrm{MoS}_{2}$ devices. The resistance for the pristine sample was $295 \mathrm{M} \Omega$, which slightly increased to $312 \mathrm{M} \Omega$ in 4 months and $355 \mathrm{M} \Omega$ in a year. Figure $6 \mathrm{~b}$ shows a histogram of resistance for all $27 \mathrm{MoS}_{2}$ samples in the chips. The average resistance of the PMMA-coated samples increased by only $6 \%$ compared to that of the pristine samples, whereas the average resistance of the unencapsulated samples increased by $430 \%$ in 4 months. In addition, the average resistance of the PMMA-coated samples increased by $40 \%$ in a year, whereas unencapsulated samples became completely insulated. The small increase of the resistance of the PMMA-coated device with time might be the effect of the moisture absorbed by PMMA because it can absorb as high as $2 \%$ of water. ${ }^{40}$ XPS measurements of the PMMA-coated 1-year-old sample show all the Mo $3 \mathrm{~d}$ and S $2 \mathrm{p}$ doublet peaks at the same BEs as the pristine sample within an error margin of $0.1 \mathrm{eV}$, and only less than $1 \% \mathrm{MoO}_{3}$ was detected (Figure $6 \mathrm{c}, \mathrm{d}$ ). These results confirm that the observed electrical property degradation of the film is due to the effect of ambient exposure and PMMA coating on the $\mathrm{MoS}_{2}$ film can effectively prevent the degradation of its electrical properties.

\section{CONCLUSIONS}

In conclusion, we report for the first time a systematic study of the electrical property variation of the $\mathrm{MoS}_{2}$ thin film under long-term ambient exposure. We demonstrated that the resistance of the $\mathrm{MoS}_{2}$ thin-film samples, prepared via the lowpressure sulfurization of the Mo film, monotonically increases with the ambient exposure time and becomes completely insulated in a year. The dual-sweep $I-V$ curve of the pristine device did not show any hysteretic behavior, while significant hysteresis in $I-V$ curves was observed for 8- and 10-month-old samples. XPS measurements reveal the gradual increase of the oxidation of the $\mathrm{MoS}_{2}$ film to form oxides of $\mathrm{Mo}$ and $\mathrm{S}$ simultaneously along with a shift of the Mo and $S$ peaks to lower 
BEs. This causes a decrease of free electron carrier in the $\mathrm{MoS}_{2}$ film, which explains the observed increase of resistance in electrical transport measurements. The localization of electrons at $\mathrm{MoO}_{3}$ domains attracts the polar molecules and moisture from atmosphere, which is the origin of the observed hysteresis in $I-V$ curves for aged samples. We also showed that the PMMA encapsulation of the $\mathrm{MoS}_{2}$ thin film can effectively prevent electrical property degradation, which shows only a $6 \%$ increase in resistance in 4 months and a $40 \%$ increase in a year.

\section{METHODS}

$\mathrm{MoS}_{2}$ Film Growth. The $\mathrm{MoS}_{2}$ thin film was grown via the low-pressure sulfurization of the molybdenum (Mo) film in a furnace equipped with a 1 in. quartz tube. A silicon substrate with a $250 \mathrm{~nm}$ thick thermal oxide capping layer was rinsed with acetone, isopropyl alcohol, and deionized water, followed by rinsing with oxygen plasma for $10 \mathrm{~min}$ to ensure the cleanliness of the substrate. The Mo film of $6 \mathrm{~nm}$ thickness was deposited on the clean $\mathrm{Si} / \mathrm{SiO}_{2}$ substrate by thermal evaporation and placed in the center of the furnace. Sulfur (S) powder (400 mg, 99.9\%, Sigma-Aldrich) was kept in a separate quartz crucible in the upstream side at a distance of $16.5 \mathrm{~cm}$ from the center of the furnace. The chamber was purged with argon (Ar) gas (99.995\% purity) for the removal of any residual oxygen and water vapor in the chamber and pumped down to a base pressure of $\sim 30$ mTorr. The flow rate of Ar gas was adjusted to 130 standard cubic centimeter per minute $(\mathrm{sccm})$ to transport $S$ vapor to the center of the furnace. The furnace was then heated to the growth temperature of $800{ }^{\circ} \mathrm{C}$ at a ramping rate of $15^{\circ} / \mathrm{min}$ and was maintained at that temperature for $55 \mathrm{~min}$. After the growth, the chamber was allowed to cool to room temperature naturally.

AFM and Raman Characterization. A tapping mode atomic force microscope (Veeco instruments, Dimension 3100) was used to determine the topography of the film. Raman characterization was performed using a WITec alpha 300 RA confocal Raman microscope with a laser source of an excitation wavelength of $532 \mathrm{~nm}$ and a power of $<1 \mathrm{~mW}$ under ambient conditions at room temperature. A $100 \times$ objective was used to focus the laser beam at a spot. Raman emission was collected and dispersed by a grating of 1800 lines-per-mm with a data accumulation duration of $3 \mathrm{~s}$.

XPS Characterization. XPS measurements of the $\mathrm{MoS}_{2}$ samples were performed using a Thermo Scientific (ESCALAB $\mathrm{Xi}$ ) XPS system with a monochromatic $\mathrm{Al} \mathrm{K} \alpha$ radiation source. A pass energy of $20 \mathrm{eV}$ with a $0.1 \mathrm{eV}$ scanning step was used for photoelectron detection. XPS spectra were taken onto the sample surface with a scan area of $300 \times 300 \mu \mathrm{m}^{2}$, and a carbon (C) $1 \mathrm{~s}$ reference line at a $\mathrm{BE}$ of $284.8 \mathrm{eV}$ was used to calibrate the charging effect.

Device Fabrication and Transport Characterization. For the electrical transport characterization of the $\mathrm{MoS}_{2}$ film, 5 $\mathrm{nm} / 40 \mathrm{~nm} \mathrm{Cr} / \mathrm{Au}$ electrodes were deposited on top of the film using a shadow mask. The drain-source electrodes were deposited with a deposition rate of $0.05 \AA$ /s at a base pressure of $5 \times 10^{-7} \mathrm{mBar}$. The electrical transport measurements of the devices were performed in a probe station using a Keithley 2400 source meter and a current preamplifier (DL instruments 1211) interfaced with the Lab View program (National Instrument). All electrical measurements were carried out at room temperature in a two-probe configuration. The devices are kept under ambient conditions (room temperature, atmospheric pressure, and humidity $51 \%$ ) and measured again for stability comparison. For PMMA encapsulation, the $\mathrm{MoS}_{2}$ devices were coated with $\sim 120 \mathrm{~nm}$ thick PMMA (molecular weight of 950k). After 4 months, PMMA was removed by dipping into acetone and electronic transport properties were measured. This was repeated for the 1-year-old samples.

\section{ASSOCIATED CONTENT}

\section{Supporting Information}

The Supporting Information is available free of charge at https://pubs.acs.org/doi/10.1021/acsomega.1c03522.

Box plot of the resistance variation of the pristine $\mathrm{MoS}_{2}$ thin film and after left under ambient conditions with and without PMMA coating, optical microscopic images of the large-area pristine $\mathrm{MoS}_{2}$ thin film and after keeping under ambient conditions, XPS O 1s spectra of pristine and ambient-exposed $\mathrm{MoS}_{2}$ films, XPS C 1s spectra of pristine and ambient-exposed $\mathrm{MoS}_{2}$ films, and AFM and SEM surface topography images of pristine and ambientexposed $\mathrm{MoS}_{2}$ thin films (PDF)

\section{AUTHOR INFORMATION}

\section{Corresponding Authors}

Bhim Chamlagain - NanoScience Technology Center and Department of Physics, University of Central Florida, Orlando, Florida 32826, United States; 10 orcid.org/0000-0002-34128323; Email: bhim.chamlagain@ucf.edu

Saiful I. Khondaker - NanoScience Technology Center and Department of Physics, University of Central Florida, Orlando, Florida 32826, United States; School of Electrical Engineering and Computer Science, University of Central Florida, Orlando, Florida 32826, United States; Email: saiful@ucf.edu

Complete contact information is available at:

https://pubs.acs.org/10.1021/acsomega.1c03522

\section{Notes}

The authors declare no competing financial interest.

\section{ACKNOWLEDGMENTS}

This work was supported by the U.S. National Science Foundation (NSF) under grant no. 1728309. We acknowledge Sajeevi Withanage and Prof. Laurene Tetard for helping with Raman measurements.

\section{REFERENCES}

(1) Gurarslan, A.; Yu, Y.; Su, L.; Yu, Y.; Suarez, F.; Yao, S.; Zhu, Y.; Ozturk, M.; Zhang, Y.; Cao, L. Surface-Energy-Assisted Perfect Transfer of Centimeter-Scale Monolayer and Few-Layer $\mathrm{MoS}_{2}$ Films onto Arbitrary Substrates. ACS Nano 2014, 8, 11522-11528.

(2) Cha, E.; Patel, M. D.; Park, J.; Hwang, J.; Prasad, V.; Cho, K.; Choi, W. $2 \mathrm{D} \mathrm{MoS}_{2}$ as an efficient protective layer for lithium metal anodes in high-performance Li-S batteries. Nat. Nanotechnol. 2018, 13, 337-344.

(3) Lin, Y.-C.; Zhang, W.; Huang, J.-K.; Liu, K.-K.; Lee, Y.-H.; Liang, C.-T.; Chu, C.-W.; Li, L.-J. Wafer-scale $\mathrm{MoS}_{2}$ thin layers prepared by $\mathrm{MoO}_{3}$ sulfurization. Nanoscale 2012, 4, 6637-6641.

(4) Vangelista, S.; Cinquanta, E.; Martella, C.; Alia, M.; Longo, M.; Lamperti, A.; Mantovan, R.; Basset, F. B.; Pezzoli, F.; Molle, A. Towards a uniform and large-scale deposition of $\mathrm{MoS}_{2}$ nanosheets via sulfurization of ultra-thin Mo-based solid films. Nanotechnology 2016, 27, 175703.

(5) Choudhary, N.; Park, J.; Hwang, J. Y.; Choi, W. Growth of largescale and thickness modulated $\mathrm{MoS}_{2}$ nanosheets. ACS Appl. Mater. Interfaces 2014, 6, 21215-21222. 
(6) Kong, D.; Wang, H.; Cha, J. J.; Pasta, M.; Koski, K. J.; Yao, J.; Cui, Y. Synthesis of $\mathrm{MoS}_{2}$ and $\mathrm{MoSe}_{2}$ films with vertically aligned layers. Nano Lett. 2013, 13, 1341-1347.

(7) Simchi, H.; Walter, T. N.; Choudhury, T. H.; Kirkley, L. Y.; Redwing, J. M.; Mohney, S. E. Sulfidation of 2D transition metals (Mo, $\mathrm{W}, \mathrm{Re}, \mathrm{Nb}, \mathrm{Ta}$ ): thermodynamics, processing, and characterization. J. Mater. Sci. 2017, 52, 10127-10139.

(8) Kim, H.-J.; Kim, H.; Yang, S.; Kwon, J.-Y. Grains in Selectively Grown $\mathrm{MoS}_{2}$ Thin Films. Small 2017, 13, 1702256.

(9) Jung, Y.; Shen, J.; Liu, Y.; Woods, J. M.; Sun, Y.; Cha, J. J. Metal seed layer thickness-induced transition from vertical to horizontal growth of $\mathrm{MoS}_{2}$ and $\mathrm{WS}_{2}$. Nano Lett. 2014, 14, 6842-6849.

(10) Choudhary, N.; Park, J.; Hwang, J. Y.; Chung, H.-S.; Dumas, K. H.; Khondaker, S. I.; Choi, W.; Jung, Y. Centimeter Scale Patterned Growth of Vertically Stacked Few Layer Only 2D $\mathrm{MoS}_{2} / \mathrm{WS}_{2}$ van der Waals Heterostructure. Sci. Rep. 2016, 6, 25456.

(11) Xue, Y.; Zhang, Y.; Liu, Y.; Liu, H.; Song, J.; Sophia, J.; Liu, J.; Xu, Z.; Xu, Q.; Wang, Z.; Zheng, J.; Liu, Y.; Li, S.; Bao, Q. Scalable Production of a Few-Layer $\mathrm{MoS}_{2} / \mathrm{WS}_{2}$ Vertical Heterojunction Array and Its Application for Photodetectors. ACS Nano 2016, 10, 573-580.

(12) Järvinen, T.; Lorite, G. S.; Peräntie, J.; Toth, G.; Saarakkala, S.; Virtanen, V. K.; Kordas, K. WS 2 and $\mathrm{MoS}_{2}$ thin film gas sensors with high response to $\mathrm{NH}_{3}$ in air at low temperature. Nanotechnology 2019, 30, 405501 .

(13) Ko, T.-J.; Wang, M.; Yoo, C.; Okogbue, E.; Islam, M. A.; Li, H.; Shawkat, M. S.; Han, S. S.; Oh, K. H.; Jung, Y. Large-area 2D TMD layers for mechanically reconfigurable electronic devices. J. Phys. D: Appl. Phys. 2020, 53, 313002.

(14) Gao, J.; Li, B.; Tan, J.; Chow, P.; Lu, T.-M.; Koratkar, N. Aging of Transition Metal Dichalcogenide Monolayers. ACS Nano 2016, 10, 2628-2635.

(15) Budania, P.; Baine, P.; Montgomery, J.; McGeough, C.; Cafolla, T.; Modreanu, M.; McNeill, D.; Mitchell, N.; Hughes, G.; Hurley, P. Long-term stability of mechanically exfoliated $\mathrm{MoS}_{2}$ flakes. MRS Commun. 2017, 7, 813-818.

(16) Yao, K.; Femi-Oyetoro, J. D.; Yao, S.; Jiang, Y.; El Bouanani, L.; Jones, D. C.; Ecton, P. A.; Philipose, U.; El Bouanani, M.; Rout, B.; Neogi, A.; Perez, J. M. Rapid ambient degradation of monolayer $\mathrm{MoS}_{2}$ after heating in air. 2D Materi. 2019, 7, 015024.

(17) Wu, J.; Li, H.; Yin, Z.; Li, H.; Liu, J.; Cao, X.; Zhang, Q.; Zhang, $\mathrm{H}$. Layer thinning and etching of mechanically exfoliated $\mathrm{MoS}_{2}$ nanosheets by thermal annealing in air. Small 2013, 9, 3314-3319.

(18) Mirabelli, G.; McGeough, C.; Schmidt, M.; McCarthy, E. K.; Monaghan, S.; Povey, I. M.; McCarthy, M.; Gity, F.; Nagle, R.; Hughes, G.; Cafolla, A.; Hurley, P. K.; Duffy, R. Air sensitivity of $\mathrm{MoS}_{2}$, $\mathrm{MoSe}_{2}$, $\mathrm{MoTe}_{2}, \mathrm{HfS}_{2}$, and HfSe 2 . J. Appl. Phys. 2016, 120, 125102.

(19) Şar, H.; Özden, A.; Demiroğlu, I.; Sevik, C.; Perkgoz, N. K.; Ay, F. Long-Term Stability Control of CVD-Grown Monolayer $\mathrm{MoS}_{2}$. Phys. Status Solidi RRL 2019, 13, 1800687.

(20) Liu, H.; Han, N.; Zhao, J. Atomistic insight into the oxidation of monolayer transition metal dichalcogenides: from structures to electronic properties. RSC Adv. 2015, 5, 17572-17581.

(21) Zhao, B.; Shang, C.; Qi, N.; Chen, Z. Y.; Chen, Z. Q. Stability of defects in monolayer $\mathrm{MoS}_{2}$ and their interaction with $\mathrm{O}_{2}$ molecule: $\mathrm{A}$ first-principles study. Appl. Surf. Sci. 2017, 412, 385-393.

(22) Martincová, J.; Otyepka, M.; Lazar, P. Is Single Layer $\mathrm{MoS}_{2}$ Stable in the Air? Chem 2017, 23, 13233-13239.

(23) Li, H.; Zhang, Q.; Yap, C. C. R.; Tay, B. K.; Edwin, T. H. T.; Olivier, A.; Baillargeat, D. From Bulk to Monolayer $\mathrm{MoS}_{2}$ : Evolution of Raman Scattering. Adv. Funct. Mater. 2012, 22, 1385-1390.

(24) Lee, C.; Yan, H.; Brus, L. E.; Heinz, T. F.; Hone, J.; Ryu, S. Anomalous lattice vibrations of single- and few-layer $\mathrm{MoS}_{2}$. ACS Nano 2010, 4, 2695-2700.

(25) Li, S.-L.; Miyazaki, H.; Song, H.; Kuramochi, H.; Nakaharai, S.; Tsukagoshi, K. Quantitative Raman spectrum and reliable thickness identification for atomic layers on insulating substrates. ACS Nano 2012, 6, 7381-7388.
(26) Baek, S. H.; Choi, Y.; Choi, W. Large-Area Growth of Uniform Single-Layer $\mathrm{MoS}_{2}$ Thin Films by Chemical Vapor Deposition. Nanoscale Res. Lett. 2015, 10, 388.

(27) Cho, D.-H.; Lee, W.-J.; Wi, J.-H.; Han, W. S.; Yun, S. J.; Shin, B.; Chung, Y.-D. Enhanced sulfurization reaction of molybdenum using a thermal cracker for forming two-dimensional $\mathrm{MoS}_{2}$ layers. Phys. Chem. Chem. Phys. 2018, 20, 16193-16201.

(28) Momose, T.; Nakamura, A.; Daniel, M.; Shimomura, M. Phosphorous doped p-type $\mathrm{MoS}_{2}$ polycrystalline thin films via direct sulfurization of Mo film. AIP Adv. 2018, 8, 025009.

(29) Islam, M. A.; Church, J.; Han, C.; Chung, H.-S.; Ji, E.; Kim, J. H.; Choudhary, N.; Lee, G.-H.; Lee, W. H.; Jung, Y. Noble metal-coated MoS2 nanofilms with vertically-aligned 2D layers for visible light-driven photocatalytic degradation of emerging water contaminants. Sci. Rep. 2017, 7, 14944.

(30) Chamlagain, B.; Withanage, S. S.; Johnston, A. C.; Khondaker, S. I. Scalable lateral heterojunction by chemical doping of $2 \mathrm{D}$ TMD thin films. Sci. Rep. 2020, 10, 12970.

(31) Late, D. J.; Liu, B.; Matte, H. S. S. R.; Dravid, V. P.; Rao, C. N. R. Hysteresis in single-layer $\mathrm{MoS}_{2}$ field effect transistors. ACS Nano 2012, 6, 5635-5641.

(32) Chamlagain, B.; Khondaker, S. I. Electrical properties tunability of large area $\mathrm{MoS}_{2}$ thin films by oxygen plasma treatment. Appl. Phys. Lett. 2020, 116, 223102.

(33) Tao, L.; Duan, X.; Wang, C.; Duan, X.; Wang, S. Plasmaengineered $\mathrm{MoS}_{2}$ thin-film as an efficient electrocatalyst for hydrogen evolution reaction. Chem. Commun. 2015, 51, 7470-7473.

(34) Neal, A. T.; Pachter, R.; Mou, S. P-type conduction in twodimensional MoS2 via oxygen incorporation. Appl. Phys. Lett. 2017, $110,193103$.

(35) Jadwiszczak, J.; O’Callaghan, C.; Zhou, Y.; Fox, D. S.; Weitz, E.; Keane, D.; Cullen, C. P.; O’Reilly, I.; Downing, C.; Shmeliov, A.; Maguire, P.; Gough, J. J.; McGuinness, C.; Ferreira, M. S.; Bradley, A. L.; Boland, J. J.; Duesberg, G. S.; Nicolosi, V.; Zhang, H. Oxidemediated recovery of field-effect mobility in plasma-treated $\mathrm{MoS}_{2}$. Sci. Adv. 2018, 4, No. eaao5031.

(36) Fan, S.; Tang, X.; Zhang, D.; Hu, X.; Liu, J.; Yang, L.; Su, J. Ambipolar and $\mathrm{n} / \mathrm{p}$-type conduction enhancement of two-dimensional materials by surface charge transfer doping. Nanoscale 2019, 11, 15359-15366.

(37) Siciliano, T.; Tepore, A.; Filippo, E.; Micocci, G.; Tepore, M. Characteristics of molybdenum trioxide nanobelts prepared by thermal evaporation technique. Mater. Chem. Phys. 2009, 114, 687-691.

(38) Afaneh, T.; Fryer, A.; Xin, Y.; Hyde, R. H.; Kapuruge, N.; Gutiérrez, H. R. Large-Area Growth and Stability of Monolayer Gallium Monochalcogenides for Optoelectronic Devices. ACS Appl. Nano Mater. 2020, 3, 7879-7887.

(39) Hong, T.; Chamlagain, B.; Lin, W.; Chuang, H.-J.; Pan, M.; Zhou, Z.; Xu, Y.-Q. Polarized photocurrent response in black phosphorus field-effect transistors. Nanoscale 2014, 6, 8978-8983.

(40) N’Diaye, M.; Pascaretti-Grizon, F.; Massin, P.; Baslé, M. F.; Chappard, D. Water absorption of poly(methyl methacrylate) measured by vertical interference microscopy. Langmuir 2012, 28, 11609-11614. 